\title{
Evaluación de recursos TIC sobre estructura atómica realizada por estudiantes de profesorado en Ciencias
}

Recibido: 15 Febrero 2014 - Revisado: 30 Abril 2014 Aceptado: 30 Mayo 2014 - Publicado: 30 Julio 2014

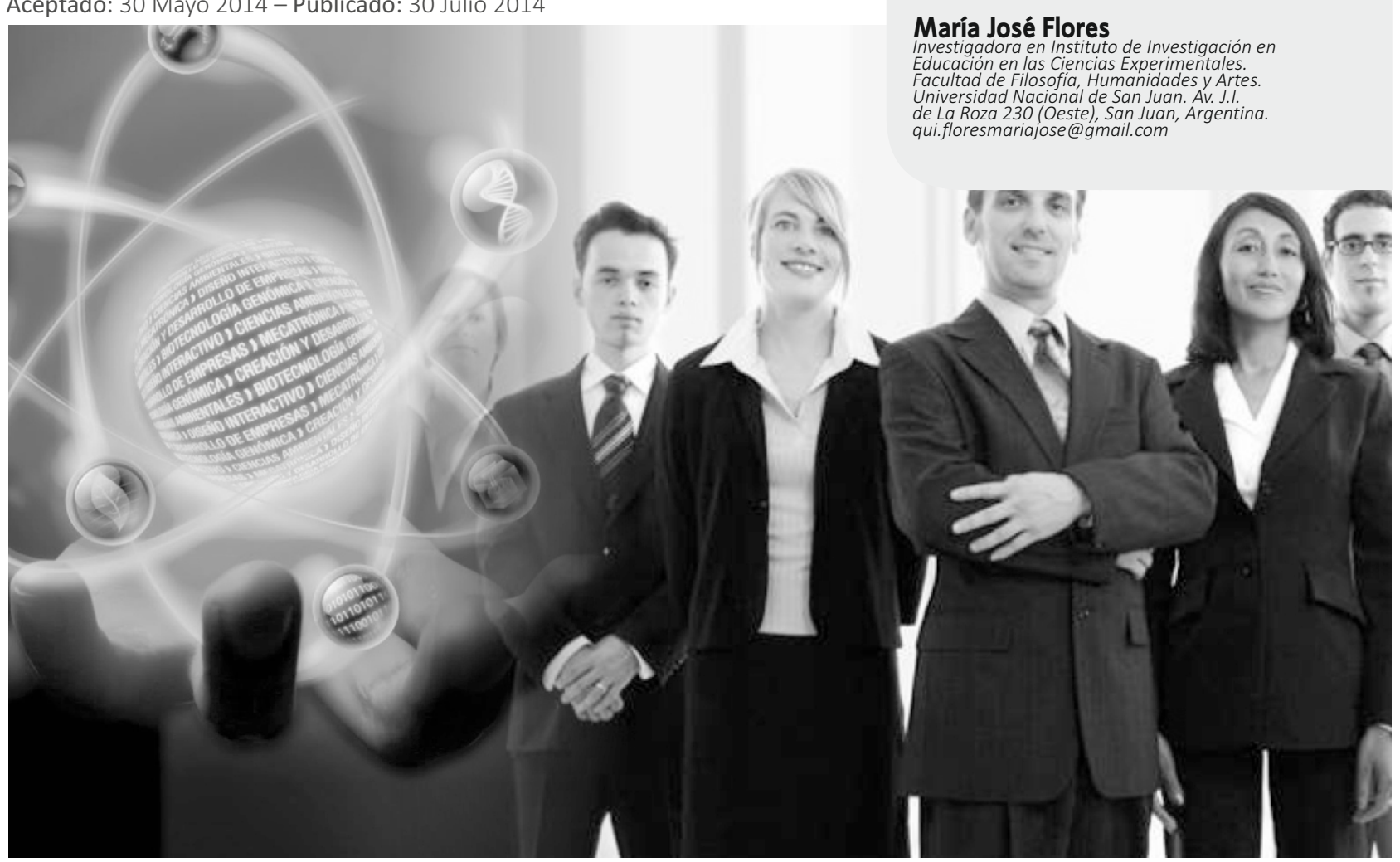

Resumen: Actualmente, se ofrecen en la web numerosos recursos educativos sobre contenidos científicos. Estos recursos pueden ser utilizados por los docentes de Ciencias en las prácticas áulicas con diferentes propósitos. En este trabajo presentamos las opiniones de estudiantes universitarios, futuros docentes de Ciencias Naturales, sobre cuatro recursos educativos gratuitos referidos a la estructura atómica. Los resultados muestran sus opiniones acerca de los mismos y los propósitos con que los utilizarían en sus futuras clases.

Palabras claves TIC; formación docente inicial; estructura atómica; evaluación

Abstract Currently, there are numerous resources about scientific content on the Web. These resources can be used by teachers of science in the classroom practices for different purposes. In this paper we present the views of some students, future teachers of Natural Sciences, about four free educational resources relating to atomic structure. The results show the purposes for these resources in their future classes and their opinions about them.

Key words TIC; initial teacher training; atomic structure; evaluation. 


\section{INTRODUCCIÓN}

Las nuevas tecnologías se presentan como herramientas eficaces para el aprendizaje de las Ciencias Naturales y su utilización genera nuevos espacios para los procesos de enseñar y de aprender, nuevos escenarios y nuevas posibilidades educativas (Coll, Mauri y Onrubia, 2008).

La manera de integrar las tecnologías de la información y la comunicación (TIC) en las clases debería trabajarse arduamente en la formación inicial, aunque hay estudios que indican que no hay una planificación de la forma adecuada de cómo debe llevarse a cabo la formación inicial y permanente del profesorado con relación al uso de las TIC (Pontes Pedrajas, 2005). Silva Quiroz et al. (2006) consideran que es urgente incorporar en los programas de formación inicial docente, elementos relacionados con la inserción de las TIC en los procesos de enseñanza y aprendizaje, que preparen a los futuros docentes para los escenarios actuales y para aquellos que se prevén para el futuro, en el corto, mediano y largo plazo. Si bien se han puesto en marcha en nuestro país programas tendientes a capacitar en este aspecto a los docentes en ejercicio, consideramos necesario realizar intervenciones en la formación inicial de estudiantes de profesorado a fin de que conozcan recursos tecnológicos educativos en el área de las Ciencias Naturales y reflexionen acerca de su utilización en las clases.

\section{ANTECEDENTES}

La existencia de recursos diversos en el área educativa hace surgir la necesidad de una evaluación crítica y muchas veces, de una adaptación por parte del docente. Dicha evaluación requiere de un conjunto de competencias para la búsqueda, identificación y jerarquización de la información y de una formación específica tanto en aspectos disciplinares como en aspectos metodológicos. Según Fainholc (2004), para un mayor aprovechamiento de los nuevos recursos tecnológicos en educación, deberían desarrollarse las capacidades para explorar eficientemente el potencial de las TIC. En la formación de los futuros profesores, esto implicaría realizar prácticas de lectura crítica en Internet con el fin de conocer las limitaciones y las posibilidades educativas de los entornos virtuales y de las simulaciones.

Un docente, hoy, debe estar preparado para adaptarse a los vertiginosos cambios sociales, culturales, económicos y tecnológicos, lo que requiere la formación de competencias específicas (Ros y Fernández 2003). Una de las competencias que Silva Quiroz et al. (2006) sugieren que se trabaje con los futuros profesores se relaciona con la evaluación de recursos, centrando la atención en las habilidades para evaluar técnica y críticamente su uso y la organización de entornos de aprendizaje.

Cuando un profesor selecciona un recurso TIC, debe realizar un proceso de evaluación y reflexión previa según las etapas de la enseñanza, los propósitos de aprendizaje y las demandas educativas del grupo de estudiantes. Estos propósitos pueden ser: motivar el aprendizaje, presentar los contenidos, dirigir las actividades de los alumnos, integrar los contenidos de aprendizaje o verificar y evaluar el rendimiento (Vargas, 1997). En este trabajo nos interesa evaluar las opiniones de estudiantes del profesorado en Física y del profesorado en Química acerca de diferentes recursos TIC a la luz de su adecuación a los mencionados propósitos.

El contenido elegido es Estructura Atómica, cuya enseñanza puede ser un desafío. La imagen del átomo planteada desde el modelo de la mecánica cuántica es complicada y muy abstracta; tan abstracta que, de hecho, las interpretaciones de la mecánica cuántica siguen siendo un tema de debate entre los físicos y los filósofos. Por otra parte, el modelo de Bohr, usado con frecuencia en las aulas, también presenta algunos problemas. Ambos modelos pueden ser útiles para 
enseñar la estructura atómica, teniendo en cuenta sus beneficios y limitaciones.

Siempre que se presenten modelos en la enseñanza, se debe tener presente y hacerle saber a los estudiantes que un modelo es una construcción imaginaria de un objeto o fenómeno (o un conjunto de ellos) que reemplaza a un aspecto de la realidad con la finalidad de realizar su estudio teórico (Adúriz Bravo, 1999; Galagovsky, 2004a y 2004b; Sánchez Blanco y Valcárcel, 2003). El uso de modelos sobre la estructura atómica permite crear una situación menos compleja que puede ser estudiada.

\section{METODOLOGÍA}

En este estudio analizamos la evaluación realizada por estudiantes de primer año del profesorado en Física y del profesorado en Química $(\mathrm{N}=27)$ para cuatro recursos TIC seleccionados referidos a la estructura atómica. La aplicación se llevó a cabo en clase de Química General, con posterioridad al tratamiento de temas relacionados con los modelos atómicos. Se dio a conocer a los estudiantes cada uno de los recursos mostrando su funcionamiento y se les pidió que evaluaran su posible utilización en distintos momentos de una clase de Ciencias Naturales.

Las pautas de evaluación de los recursos se basaron en la aplicabilidad del mismo para motivar, aprender y/o evaluar a los alumnos. Para analizar las propuestas, no les proporcionamos criterios específicos mediante los cuales debían hacerlo, sino la siguiente pregunta semiestructurada cuya respuesta debían justificar: “¿Utilizarías esta propuesta para: a) interesar a los alumnos en el estudio de la estructura atómica; b) que los alumnos aprendan sobre la estructura atómica y/o c) evaluar los aprendizajes de los alumnos sobre la estructura atómica?". Así, los estudiantes evaluaron las alternativas en base a consideraciones personales. Las respuestas se recabaron en forma escrita e individual.
Las propuestas que analizaron los estudiantes fueron las siguientes:

Propuesta 1: Apunte que contiene información acerca de la estructura atómica. Incluye el tratamiento de las nociones de átomo, partículas subatómicas, núcleo, unidad de masa atómica, número atómico, número másico, isótopos, isóbaros, anión y catión. El texto presentado incluye definiciones y ejemplos que están acompañados de dibujos que representan la estructura del átomo.

Link de acceso:

http://es.scribd.com/doc/6162187/Apuntes-2Estructura-Atomica

Propuesta 2: Propuesta de ejercicios extraída de una página de una cátedra universitaria de Química. Consta de ocho ejercicios con opción múltiple, cuyas respuestas correctas se incluyen al final del archivo.

Link de acceso:

http://www.ub.edu.ar/catedras/ingenieria/quimica_ general/temas/ejercicios_de_estructura_atomica_ _2_).doc

Propuesta 3: Recurso que incluye una tabla periódica interactiva que permite al usuario pulsar sobre el símbolo de un elemento para obtener su configuración electrónica. Puede también hacer girar los electrones, según el modelo atómico de Bohr.

Link de acceso:

http://www.deciencias.net/proyectos/4particulares/ quimica/atomo/comp/estructura.htm

Propuesta 4: Simulación interactiva que permite construir un átomo con protones, neutrones y electrones, y ver cómo cambian el elemento, la carga y la masa. Incluye además un juego interactivo en el que se aplican los contenidos involucrados en la simulación seleccionada que otorga puntajes a partir de las respuestas dadas.

Link de acceso:

http://phet.colorado.edu/en/simulations/translated/ es 
Las respuestas de los estudiantes fueron analizadas teniendo en cuenta las categorías "Interesar", "Aprender" y "Evaluar" y las justificaciones propuestas por ellos se estudiaron cualitativamente teniendo en cuenta los comentarios y apreciaciones acerca de la posible implementación del recurso.

\section{RESULTADOSY DISCUSIÓN}

Los estudiantes interpretaron que cada una de las propuestas podría utilizarse con diversos objetivos. En la Fig. 1, se observa la cantidad de alumnos que consideró adecuada cada una de las propuestas para Interesar, Aprender y Evaluar, respectivamente.

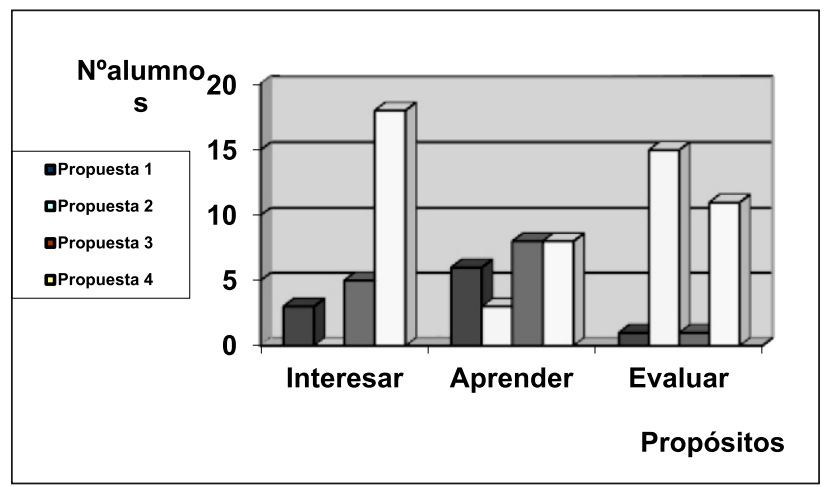

Figura 1. Número de alumnos en función de los propósitos con que pueden utilizarse las propuestas.

Consideramos importante destacar los siguientes resultados:

- El recurso interactivo presentado como Propuesta 4 es seleccionado preferentemente para interesar a los estudiantes, mientras que consideran que la 2 no es útil para este fin. Los comentarios que justifican estas respuestas se refieren especialmente a que la Propuesta 4 es didáctica y entretenida, sirve como refuerzo interactivo; los juegos e imágenes con movimiento atraen más la atención y al incentivar la competencia en el juego, permite que los estudiantes se esfuercen.
- Las cuatro propuestas han sido seleccionadas como aptas para aprender, aun cuando presentan los contenidos de formas diversas. Las cualidades que los estudiantes destacan de cada una son: la Propuesta 1 contiene todo el detalle, las definiciones y ejemplos y presenta en forma escrita los contenidos; la Propuesta 2 permite razonar y recordar cómo es la estructura de un átomo y al resolver los ejercicios, se utilizan y se fijan los conceptos; la Propuesta 3 presenta el modelo de estructura atómica e incluye una gráfica en colores que genera una dinámica que favorece el aprendizaje y la Propuesta 4 permite manipular el programa para aprender y "construir un átomo" partiendo de protones, electrones y neutrones y visualizarlo en forma gráfica.

- Las propuestas 2 y 4 , basadas en ejercicios de aplicación, son consideradas adecuadas para evaluar. La elección de la Propuesta 2 responde a que permite pensar y razonar para responder por lo que ayuda al docente a detectar si saben o no; es lo más parecido a un examen y ayuda a poner en práctica todo el conocimiento aprendido. De la Propuesta 4, destacan que es interactiva y gráfica y ayuda a ejercitar lo aprendido mediante el juego.

- Los alumnos tienen mayor preferencia por la Propuesta 4 y es elegida para los tres propósitos, lo que evidencia también la preferencia por un recurso TIC basado en una simulación interactiva.

\section{RESULTADOSY DISCUSIÓN}

Los resultados muestran que un mismo recurso puede utilizarse en diferentes momentos y con distintos objetivos en los procesos de enseñar y de aprender Ciencias Naturales, dependiendo del grupo de estudiantes y del contexto. Además, en la justificación de las selecciones quedó manifestada la capacidad de los estudiantes para realizar una lectura crítica de las propuestas presentadas. Por este motivo es importante que en la formación inicial de los futuros profesores se proponga este tipo de actividad y que se 
los prepare para evaluar la adecuación de cada recurso TIC, determinando los momentos de la secuencia didáctica apropiados para aplicarlos y explicitando los propósitos con que se los usa.

Esta investigación puede ser un muy buen punto de partida para un estudio más profundo en la formación inicial de los profesores, en cuanto a la evaluación crítica y selección de recursos TICs para uso en el aula. Para que la selección, la implementación y la evaluación de la eficacia de los recursos sean apropiadas, deberían conjugarse en la formación del docente, aspectos disciplinares, didácticos y tecnológicos y la reflexión sobre la propia práctica para hacer las adecuaciones que sean necesarias. Una vez disponible el recurso, los profesores, con el conocimiento disciplinar adecuado, podrán ayudar al estudiante a interpretar los contenidos. Con el conocimiento didáctico, propondrán actividades que favorezcan la comprensión y con el conocimiento tecnológico, sabrán buscar, adaptar y utilizar los recursos TIC para trabajarlos en las clases. Para hacer la selección y la adaptación de los contenidos y las actividades, deberán tener en cuenta el contexto según los criterios que su formación y experiencia les haga considerar buenos para cada instancia en que deban enseñar.

AGRADECIMIENTOS A la Universidad Nacional de San Juan (Argentina), que subsidia las investigaciones realizadas.

\section{REFERENCIAS}

Adúriz-Bravo, A. (1999). Elementos de teoría y de campo para la construcción de un análisis epistemológico de la didáctica de las ciencias. Tesis de maestría. Departament de Didáctica de las Ciències i la Matemática. Universitat Autónoma de Barcelona.

Coll, C., Mauri T. y Onrubia, J. (2008). Los entornos virtuales de aprendizaje basados en el análisis de casos y la resolución de problemas. En Coll, C. y Monereo, C. (Eds). Psicología de la educación virtual. Aprender y enseñar con las Tecnologías de la Información y la Comunicación. Madrid: Editorial Morata, 213-222.Fainholc, B. (2004). Lectura crítica en Internet. Análisis y utilización de los recursos tecnológicos en educación. Santa Fe (Argentina): Homo Sapiens Ediciones.

Galagosky, L. R. (2004a). Del aprendizaje significativo al aprendizaje sustentable. Parte 1: El modelo teórico. Enseñanza de las Ciencias, 22(2), 229-240.

Galagosky, L. R. (2004b). Del aprendizaje significativo al aprendizaje sustentable. Parte 2: Derivaciones comunicacionales y didácticas. Enseñanza de las Ciencias, 22(3), 349-364.

Pontes Pedrajas, A. (2005). Aplicaciones de las tecnologías de la información y de la comunicación en la educación científica. Primera parte: funciones y recursos. Revista Eureka sobre Enseñanza y Divulgación de las Ciencias, 2(1), 2-18.

Ros, N. y Fernández, G. (2003). Formación y capacitación docente continua: la producción de material didáctico para la modalidad de educación a distancia. Revista Iberoamericana de Educación, Experiencias e Innovaciones. Disponible en: http://www.rieoei.org/experiencias64.htm

Sánchez Blanco, G. y Valcárcel, M.V. (2003). Los modelos en la enseñanza de la química: concepto de 


\section{Ingenierías:":ra}

sustancia pura. Alambique Didáctica de las Ciencias Experimentales, 5, 45-59.

Silva Quiroz, J., Gros Salvat, B., Rodríguez, J. y Garrido, J.M. (2006). Estándares en tecnologías de la información y la comunicación para la formación inicial docente situación actual y el caso chileno. Revista Iberoamericana de Educación, 38(3). Disponible en: http://www.rieoei.org/1391.htm

Vargas, E. A. (1997). Metodología de la enseñanza de las Ciencias Naturales. Antología. San José de Costa Rica: UNED. 